\title{
A putative pyruvate transporter TaBASS2 positively regulates salinity tolerance in wheat via modulation of $A B / 4$ expression
}

Yang Zhao ${ }^{1 \dagger}$, Xinghui $\mathrm{Ai}^{1+}$, Mengcheng Wang ${ }^{1}$, Langtao Xiao ${ }^{2}$ and Guangmin Xia ${ }^{{ }^{*}}$

\begin{abstract}
Background: High salinity adversely affects crop production. Pyruvic acid is the precursor of abscisic acid (ABA) and other chemicals that are synthesized in chloroplast, some of which are involved in the response to salt. The transportation of pyruvic acid into chloroplast is mediated by pyruvate transporters. However, whether pyruvate transporters are involved in salt response has not been studied so far. Here, we answered this issue by assessing the function of a wheat pyruvate transporter in salt response.

Results: A pyruvate transporter TaBASS2 was isolated from salt-tolerant wheat cultivar Shanrong 3. The expression of TaBASS2 was induced by $\mathrm{NaCl}$ stress as well as $\mathrm{H}_{2} \mathrm{O}_{2}$ and ABA treatments. Constitutive expression of TaBASS2 in Arabidopsis bass2-1 mutant complemented the mevastatin-sensitive phenotype that reflects the deficiency of transporting pyruvic acid into chloroplast. Overexpression of TaBASS2 enhanced salinity tolerance and reactive oxygen species scavenging in wheat. Arabidopsis constitutively expressing TaBASS2 also exhibited enhanced tolerance to salinity and oxidative stress. In Arabidopsis, TaBASS2 repressed the expression of ABA INSENSITIVE 4 $(A B / 4)$, a node linking ABA signaling and plastid retrograde signaling pathways. However, the enhanced salinity tolerance of TaBASS2 overexpression Arabidopsis was abolished when AB/4 expression was restored to the level of wild-type through overexpressing $A B / 4$.
\end{abstract}

Conclusions: Our work demonstrates that TaBASS2 enhances salinity tolerance of plants via modulating ABI4 expression. This indicates that pyruvate transporters indeed participate in the interaction of plants with environmental stimuli.

Keywords: ABI4, BASS2, Oxidative stress, ROS, Salinity tolerance, Wheat

\section{Background}

High levels of soil salinity impose osmotic stress and ion toxicity on plants, leading to cell damage and growth arrest. Most crop plants are sensitive to excess salt concentration in soil [1]. With the salt-affected farming land expanding nowadays (http://www.fao.org/soils-portal/ soil-management/management-of-some-problemsoils/saltaffected-soils/more-information-on-salt-affected-sovils/en/), salinity stress has become one of the most serious limiting factors in crop production. As an urgent global challenge of

\footnotetext{
* Correspondence: xiagm@sdu.edu.cn

${ }^{\dagger}$ Equal contributors

${ }^{1}$ The Key Laboratory of Plant Cell Engineering and Germplasm Innovation, Ministry of Education, School of Life Science, Shandong University, 27

Shanda South Road, Jinan, Shandong 250100, China

Full list of author information is available at the end of the article
}

food security occurs, it is of great importance to understand the mechanism underlying plant response to salinity stress and develop novel salinity tolerant crop cultivars.

In the past few decades, extensive studies on salinity stress in plants, especially in the model plant Arabidopsis thaliana, have uncovered a number of genes involved in plant salt tolerance [2, 3]. Both abscisic acid (ABA)dependent and -independent signaling pathways play important roles during this process $[4,5]$. ABA INSENSITIVE 4 (ABI4) is an AP2/EREBP transcription factor that functions as a positive regulator in the ABA signaling pathway during seed development and germination [6]. ABI4 participates in other aspects of plant development, including salinity response and retrograde signaling [7-11]. Under salinity stress, three loss-of-function mutations in $A B I 4$ conferred increased tolerance in both seedling and 
adult stage, while the transgenic plants overexpressing $A B I 4$ were hypersensitive to $\mathrm{NaCl}$ treatment $[7,11]$. Further studies revealed that $\mathrm{ABI} 4$ negatively regulated the expression of a high affinity $\mathrm{K}^{+}$transporter, HKT1;1, by directly binding to two ABI4-binding elements (ABE) in HKT1;1's promoter region [11].

Besides osmotic stress and ion toxicity, high salinity condition also adversely affects photosynthesis, cellular energy depletion and redox homeostasis [12-14]. Production and accumulation of excess reactive oxygen species (ROS), such as superoxide $\left(\mathrm{O}_{2}^{-}\right)$and hydrogen peroxide $\left(\mathrm{H}_{2} \mathrm{O}_{2}\right)$, cause oxidative damages in apoplastic compartments and cellular membranes. ROS also function as signaling molecules to activate gene expression in nucleus $[1,15,16]$, including a number of ROS scavenging genes, such as CATALASE (CAT) $[17,18]$. In $A$. thaliana, a transient burst of ROS production follows the imposition of abiotic stresses, and any disruption to ROS synthesis has a negative effect on the plant's growth and its ability in stress response [13].

Chloroplast is closely associated with salt response in plants. It is not only a factory for energy assimilation but also the site for synthesis of ABA and other important metabolites, for pyruvic acid serves as the precursor. The bile acid/sodium symporter 2 (BASS2) is responsible for pyruvate uptake into chloroplast [19]. In Arabidopsis bass 2-1 mutant, the plastidal isopentenyl diphosphate (IPP) synthesis is blocked, so the mutant seedlings exhibit increased sensitivity to mevastatin, an inhibitor of cytosolic IPP synthesis pathway [19].

As an important staple crop, bread wheat belongs to glycophytes and displays high sensitivity to excess soil salinity, while its halophytic relative tall wheatgrass (Thinopyrum ponticum) is able to grow at salt concentrations as high as in seawater. In our previous study, a salinity-tolerant introgression wheat cultivar Shanrong No. 3 (SR3) was bred using asymmetric somatic hybridization [20]. SR3 wheat plants exhibited high level of tolerance under osmotic and saline stresses, and better performance in removal of toxic substances [21, 22]. In a further transcriptomic study, we identified a putative pyruvate transporter gene, TaBASS2, was up-regulated by $\mathrm{NaCl}$ treatment in SR3 instead of salinity-sensitive JN177 [23]. Here, we isolated the TaBASS2 sequence and characterized its role in salinity tolerance. Constitutively expressing TaBASS2 enhanced the salinity tolerance in transgenic wheat and Arabidopsis. The ROS contents and scavenging activity were enhanced in the transgenic plants as well. Further experiments indicated TaBASS2 positively regulates plant response to salinity stress by repressing $A B I 4$ expression.

\section{Results}

TaBASS2 is induced by $\mathrm{NaCl}$ treatment

To determine the expression pattern of TaBASS2 under salinity stress, three-leaf-stage SR3 seedlings were treated with $200 \mathrm{mM} \mathrm{NaCl}$ solution and monitored TaBASS2 expression in roots. As shown in Fig. 1a, TaBASS2 was induced by two-fold as early as $1 \mathrm{~h}$ of the treatment, and the expression level increased up to four-fold at $24 \mathrm{~h}$. The treatment with $\mathrm{H}_{2} \mathrm{O}_{2}$ or ABA also resulted in a similar induction of TaBASS2 expression at $24 \mathrm{~h}$ (Fig. 1b, c). These results demonstrated that TaBASS2 expression responded to high level of salinity as well as other stress signals. Various tissue types were also collected for another expression assay. The result showed that TaBASS2 was transcribed in all the tested tissues, with higher expression levels in the green tissues (Fig. 1d).

To study the biological role of TaBASS2 in plant response to salinity stress, the coding sequence (CDS) was cloned from a cDNA library constructed from the $\mathrm{NaCl}$ treated SR3 seedlings. The resulting CDS was 1,242 bp in length, encoding a 413-aa putative BASS protein with eight transmembrane domains. The NCBI non-redundant protein sequence database was searched for the homologues, and 42 similar proteins across various organisms were found. The multi-alignment with five closest homologs showed that the most conserved regions resided in the eight transmembrane domains (indicated as TM1-TM8 in Additional file 1). A phylogenetic analysis clustered the wheat BASS amino acid sequence with those from monocots. The sequences from dicotyledonous species, including BASS2 (At2g26900; Additional file 1), clustered together, which together with the monocotyledonous cluster formed a BASS2 clade. Since the wheat BASS protein sequence shared a high similarity with BASS2, the gene was therefore named as TaBASS2, which was the first BASS gene cloned in bread wheat.

\section{TaBASS2 complements Arabidopsis bass2-1 mutant}

Arabidopsis BASS2 is a sodium-dependent pyruvate transporter localized in plastid. Its knockout mutant in Arabidopsis, bass2-1, displays increased sensitivity to mevastatin [19]. To determine whether TaBASS2 has the same function as BASS2, the Arabidopsis bass2-1 mutant was transformed with a 35S::TaBASS2 construct. Two transgenic lines with high TaBASS2 expression levels, bass21;35S::TaBASS2 \#1 and \#2, were selected to test the sensitivity to mevastatin (Fig. 2b). After a ten-day treatment with $500 \mathrm{nM}$ mevastatin, the bass2-1 seedlings showed severe abnormality in cotyledon, while the bass2-1;35S::TaBASS2 seedlings developed a normal phenotype, the same as the wild-type Arabidopsis (Fig. 2a, c). This result demonstrated that TaBASS 2 completely complemented the mevastatinsensitive phenotype of bass2-1. Moreover, the TaBASS2GFP fusion protein was localized in plastid when transiently expressed in Arabidopsis mesophyll protoplasts (Additional file 2). These results together indicate that TaBASS2 is a genuine homolog of BASS2, which functions as a pyruvate transporter. 

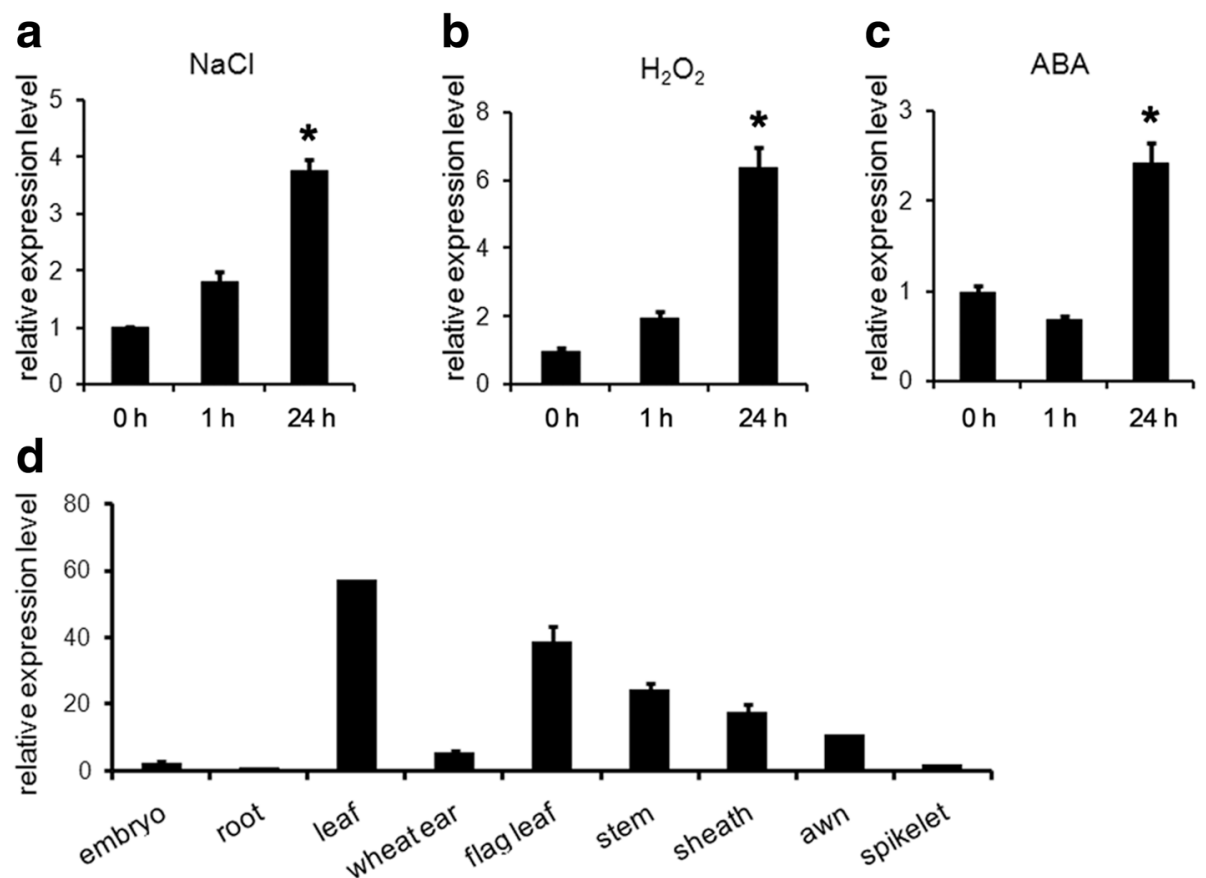

Fig. 1 TaBASS2 expression in wheat cv. SR3 plants treated with (a) $200 \mathrm{mM} \mathrm{NaCl}$, (b) $100 \mu \mathrm{M} \mathrm{ABA}$ or (c) $10 \mathrm{mM} \mathrm{H}_{2} \mathrm{O}_{2}$, and in different tissues (d). Error bars represent the standard errors $(n=3)$, with each replicate comprising at least 12 plants. Columns labeled with an asterisk in (a-c) indicate significant differences from those at $0 \mathrm{~h}(P<0.05, t$-test). The expression levels were determined by RT-qPCR using TaCyclophilin as the internal control
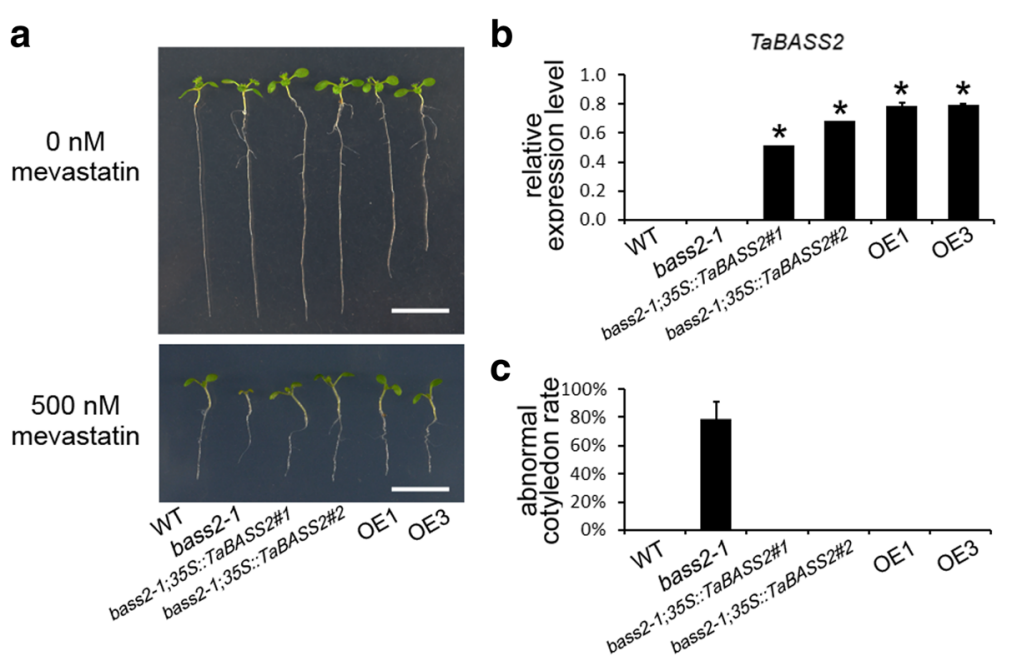

Fig. 2 TaBASS2 complements the bass2-1 mutant. a The wild-type, bass2-1, bass2-1;35S::TaBASS2 (\#1 and \#2), and 355::TaBASS2 (OE1 and OE3) plants after a seven-day exposure to $500 \mathrm{nM}$ mevastatin. Bar $=1 \mathrm{~cm}$. b TaBASS2 expression in the wild-type, bass2-1, bass2-1;355::TaBASS2 and 35S::TaBASS2 lines. The expression levels were determined by RT-qPCR using AtACT2 as the internal control. c The percentage of seedlings with abnormal cotyledons in the wild-type, bass2-1, bass2-1;35S:: TaBASS2 and 35S:: TaBASS2 lines. Error bars in (b, c) represent the standard errors $(n=3)$, with each replicate comprising at least 12 plants; columns labeled with an asterisk indicate means differing significantly from bass $2-1$ 


\section{Overexpressing TaBASS2 enhanced salinity tolerance in wheat seedlings}

The induction of TaBASS 2 by the $\mathrm{NaCl}$ treatment suggests its role in plant response to salinity stress (Fig. 1a). To characterize its function in wheat, TaBASS2 was overexpressed in a salinity sensitive wheat cultivar YM20. From 34 independent transgenic lines, two lines (OX1 and OX21) with high transgene expression were selected for further experiments (Fig. 3c). Both OX1 and OX21 transgenic lines developed shorter shoots and roots than the empty vector control (VC) seedlings (Additional file 3). Thus, to determine the extent of salinity tolerance, the shoot/root lengths of $\mathrm{NaCl}$-treated plants were normalized by dividing the shoot/root lengths of untreated plants to get relative shoot/root growth rates. After an eight-day $\mathrm{NaCl}$ treatment, the relative shoot growth in the $\mathrm{VC}$ line was around $72 \%$ of that under the control condition, and the relative root growth was around $85 \%$ compared with that under the control condition, displaying suppression in plant growth under high $\mathrm{NaCl}$ concentration. However, in the NaCl-treated OX1 and OX21 plants, both the shoot and root growth showed less suppression compared with the VC plants. After the $\mathrm{NaCl}$ treatment, the relative shoot growths were $98 \%$ and $95 \%$ compared with the control in OX1 and OX21, respectively, and the relative root growths were $90 \%$ and $91 \%$ of those under the control condition (Fig. 3d, e). These phenotypic results demonstrated that constitutively expressing TaBASS2 relieved the growth suppression imposed by high saline concentration, thus enhanced salinity tolerance in the transgenic wheat plants.
The constitutive expression of TaBASS2 in A. thaliana enhanced the salinity tolerance

To further reveal its biological role in salinity tolerance, transgenic Arabidopsis plants constitutively expressing TaBASS2 driven by a $35 \mathrm{~S}$ promoter were generated, from which two independent lines with high TaBASS2 expression levels, OE1 and OE3, were selected for further experiments (Fig. 2b). The wild-type and transgenic lines were treated with a series concentrations of $\mathrm{NaCl}$ to determine their salinity response. Given that the roots of transgenic Arabidopsis seedlings were shorter than those in the wild-type (Fig. 4a, Additional file 4), the relative root growth rate was calculated to determine their salinity tolerance. Under a $50 \mathrm{mM} \mathrm{NaCl}$ treatment, the relative root growth rates of wild-type plants were $53 \%$ of those under the control condition, while the relative root growth rates in the both transgenic plants were $73 \%$ of those under the control condition (Fig. 4b, e). When the $\mathrm{NaCl}$ concentration was raised to $100 \mathrm{mM}$, the relative root growth in the wild-type was $27 \%$, but $47 \%$ and $33 \%$ in OE1 and OE3, respectively (Fig. 4c, e); under the $125 \mathrm{mM} \mathrm{NaCl}$ treatment, the relative root growth was about 9, 29 and $20 \%$ in the wild-type and the two OE lines, respectively (Fig. 4d, e). This result demonstrated the Arabidopsis seedlings with constitutive expression of TaBASS2 exhibited enhanced tolerance to salinity stress.

A further experiment was carried out to determine the salinity tolerance in the adult transgenic plants constitutively expressing TaBASS2. Four-week-old soil-grown wildtype and two 35S::TaBASS2 lines were treated with
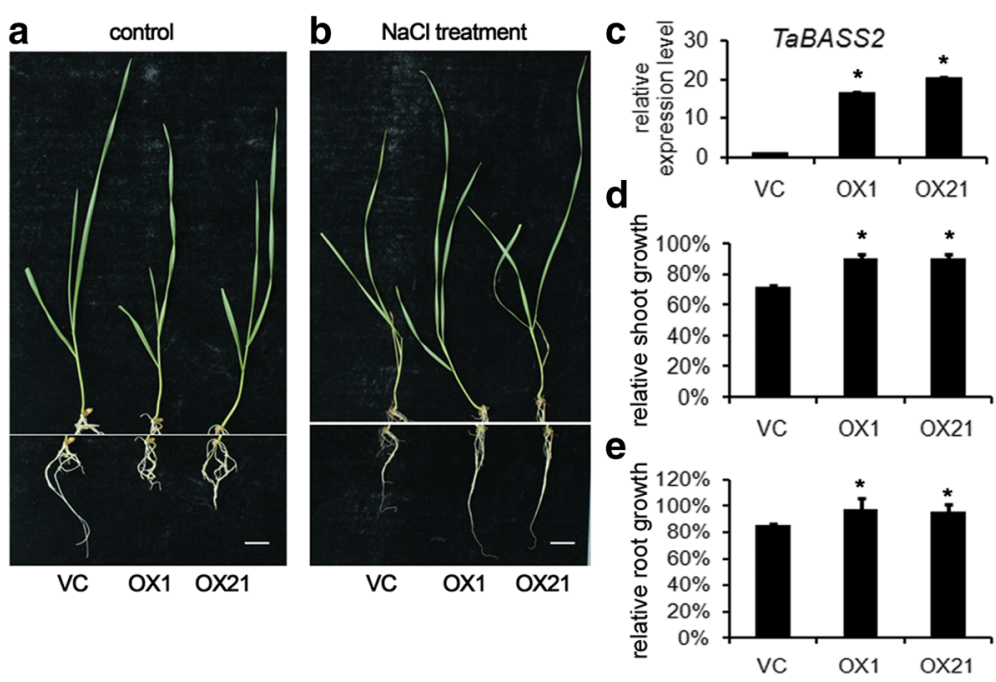

Fig. 3 Constitutively expressing TaBASS2 enhances salinity tolerance in wheat. $\mathbf{a}$, b The vector control (VC) and two Ubi::TaBASS2 wheat seedlings (OX1 and OX21) after an eight-day mock or NaCl treatment. Bar $=1 \mathrm{~cm}$. c TaBASS2 expression in the genotypes listed in (a). The expression levels were determined by RT-qPCR using TaCyclophilin in wheat as the internal control. Error bars represent the standard errors $(n=3)$, with each replicate comprising at least 12 plants. $\mathbf{d}$, e Relative shoot and root growth in the genotypes listed in (a). Error bars represent the standard errors $(n=3)$, with each replicate comprising at least 30 plants. Columns labeled with an asterisk indicate means differing significantly from the VC result $(P<0.05, t$-test $)$ 
a

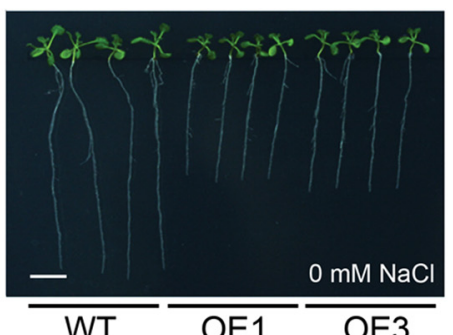

C

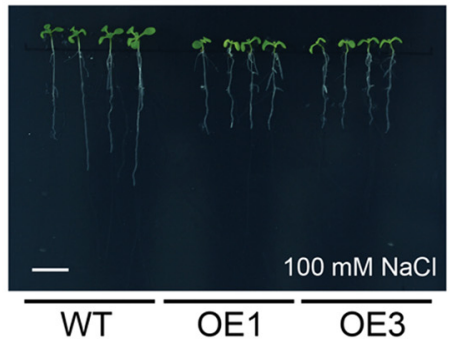

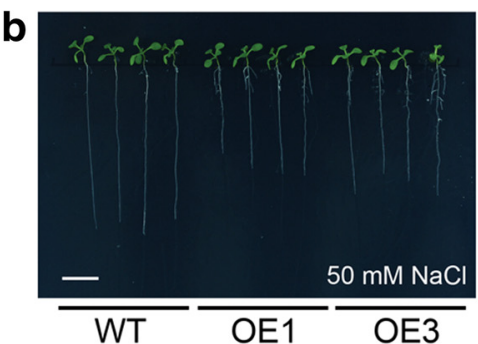

d

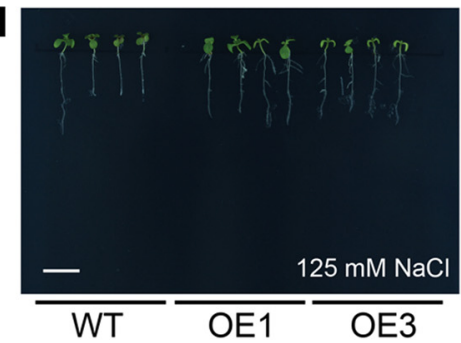

e

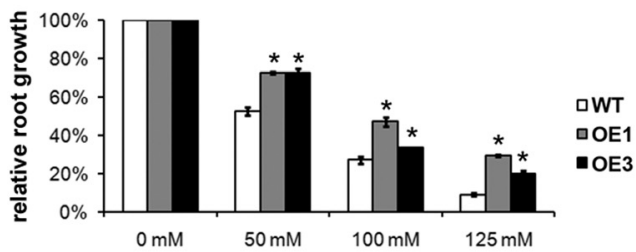

$\mathbf{f}$

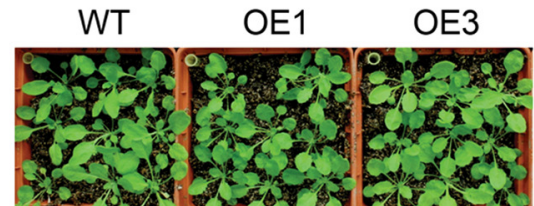

$10 \mathrm{~d}$

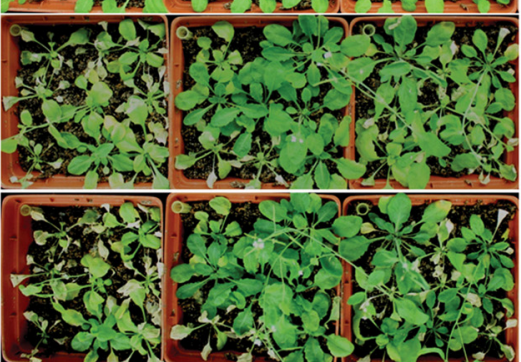

g

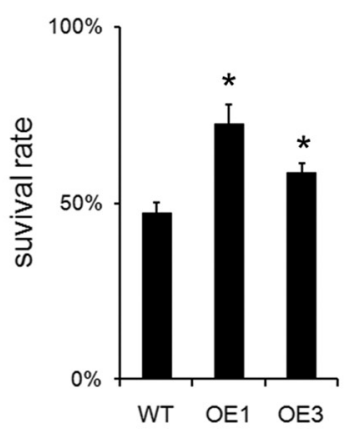

Fig. 4 Constitutively expressing TaBASS2 enhances the salinity tolerance in Arabidopsis. a-d The wild-type seedlings and two 35S::TaBASS2 transgenic lines (OE1 and OE3) after a ten-day treatment with 0, 50, 100 or $125 \mathrm{mM} \mathrm{NaCl}$. Bar $=1 \mathrm{~cm}$. e Relative root growth of the wild-type and OE plants treated with 0,50,100 or $125 \mathrm{mM} \mathrm{NaCl}$. f The four-week-old soil-grown wild-type and OE plants 0,10 and 14 days after $\mathrm{NaCl}$ treatment. $\mathbf{g}$ Plant survival rates measured at 14 day after $\mathrm{NaCl}$ treatment. Error bars in $(\mathbf{e}, \mathbf{g})$ represent the standard errors $(n=3)$, with each replicate comprising at least 30 plants. Columns labeled with an asterisk indicate means differing significantly from the $W T$ result $(P<0.05, t$-test)

increasing concentration of $\mathrm{NaCl}$ for 14 days, and the survival rates were scored at the 14th day following the treatment (See Methods). As shown in Fig. 4f, after a tenday $\mathrm{NaCl}$ treatment, the leaves of wild-type plants developed chlorosis, while the leaves of OE plants showed less severe response. At the 14th day after treatment, more than half of the wild-type plants died of high levels of soil salinity, while the majority of $\mathrm{OE}$ plants kept alive and even grew bigger (Fig. 4f). Consistent with the phenotypic response, the survival rate was $47 \%$ in the wild-type plants at the 14th day after treatment, while it was $72 \%$ and $58 \%$ in the OE1 and OE3 lines respectively, significantly higher than that in the wild-type (Fig. 4f, g). It is also interesting to mention that bass2-1 mutant showed no difference in the survival rate under $\mathrm{NaCl}$ treatment, probably due to the cytosolic IPP pathway (Additional file 5). These results showed that the 35S::TaBASS2 transgenic plants exhibited enhanced tolerance to $\mathrm{NaCl}$ treatment in both seedling and adult stages, demonstrating that constitutive expression of TaBASS2 enhanced salinity tolerance in Arabidopsis. 
The transgenic Arabidopsis constitutively expressing TaBASS2 showed the enhanced oxidative tolerance ROS is involved in plant response to salinity stress. To determine the role of TaBASS2 in oxidative stress response, the 35S::TaBASS2 Arabidposis plant were assessed under $\mathrm{H}_{2} \mathrm{O}_{2}$ treatment. As shown in Fig. 5a, b and d, under the treatment of $1 \mathrm{mM} \mathrm{H}_{2} \mathrm{O}_{2}$, the relative root growth rate of the wild-type plants was $25 \%$ of that under the control condition, significantly smaller than the relative root growth rates ( $45 \%$ and $35 \%$ ) of OE1 and OE3, respectively. When the $\mathrm{H}_{2} \mathrm{O}_{2}$ concentration was raised to $1.5 \mathrm{mM}$, the relative root growth rates were 22,40 and $29 \%$ in the wild-type, OE1 and OE3 seedlings, respectively (Fig. 5a, c, d). These results demonstrated that the transgenic Arabidopsis plants constitutively expressing TaBASS2 had higher tolerance to oxidative stress than the wild-type plants did. Moreover, treatment with methyl viologen (MV), which generates superoxide anions in plastids, demonstrated that these OE lines also displayed enhanced tolerance to plastidial oxidative stress (Additional file 6).
DAB staining results showed that both Arabidopsis OE lines had higher $\mathrm{H}_{2} \mathrm{O}_{2}$ levels in vivo than the wild-type plants (Fig. 5e). And the expression levels of the ROSscavenging catalase 1 (CAT1) were also constitutively up-regulated in the transgenic Arabidopsis plants constitutively expressing TaBASS2 (Fig. 5f), along with enhanced CAT1 enzyme activity (Fig. $5 \mathrm{~g}$ ). These results demonstrated that the constitutive expression of TaBASS2 led to an increase in ROS content and ROS-scavenging activity in the transgenic Arabidopsis plants, suggesting constitutive activation of ROS signaling.

The enhanced salinity tolerance in transgenic Arabidopsis expressing TaBASS2 was achieved through repressing $A B I 4$ expression

ABA plays an important role in response to salinity stress, so the relationship between ABA signaling and the enhanced salinity tolerance was investigated in the transgenic plants constitutively expressing TaBASS2. Firstly, we found the endogenous ABA contents were
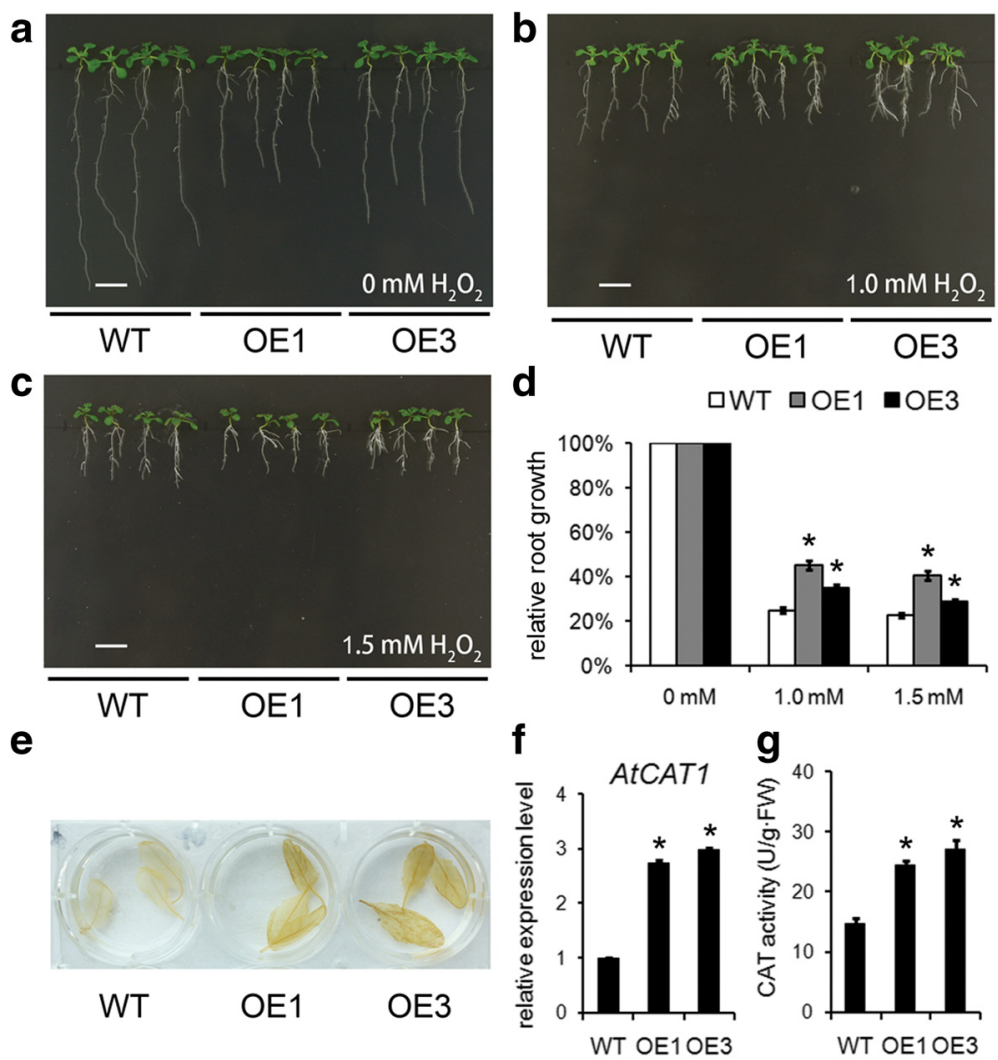

Fig. 5 Constitutively expressing TaBASS2 enhances ROS content and ROS scavenging activity. a-c The wild-type seedlings and two 35S::TaBASS2 transgenic lines (OE1 and OE3) after a ten-day treatment with 0,1 or $1.5 \mathrm{mM} \mathrm{H}_{2} \mathrm{O}_{2}$. Bar $=1 \mathrm{~cm}$. d Relative root growth of the wild-type and OE plants treated with 0,1 or $1.5 \mathrm{mM} \mathrm{H}_{2} \mathrm{O}_{2}$. e DAB staining of the leaves from four-week-old soil-grown wild-type and OE plants. $\mathbf{f}$ The expression levels of AtCAT1 in 12-day-old wild-type and OE seedlings. $\mathbf{g}$ The catalase activity in 12-day-old wild-type and OE seedlings. Error bars in (d) represent the standard errors $(n=3)$, with each replicate comprising 30 seedlings. Error bars in $(\mathbf{f}, \mathbf{g})$ represent the standard errors $(n=3)$, with each replicate comprising at least 12 plants. Columns labeled with an asterisk indicate means differing significantly from the WT result $(P<0.05, t$-test). The expression levels were determined by RT-qPCR using AtACT2 in Arabidopsis as the internal control 
comparable among the wild-type Arabidopsis and 35S::TaBASS2 transgenic plants (Additional file 7). The expression levels of genes at the downstream of ABA-dependent stress responsive pathway were also assessed. Most of these genes, including RESPONSIVE TO DESICCATION 29A (RD29A), RD29B, RD22 and $M Y B 2$, showed no difference in their expression levels between the wild-type and 35S::TaBASS2 plants (Additional file 8a-d). On the contrast, the expression of $A B I 4$, a key component of ABA signaling pathway, was reduced by more than one fold in the $35 S:: T a B A S S 2$ transgenic lines compared to the wild-type (Fig. 6a). These results demonstrated that constitutively expressing TaBASS2 repressed ABI4 expression without affecting ABA biosynthesis. Furthermore, the expression levels of $H K T 1$, the high affinity $\mathrm{K}^{+}$transporter directly regulated by ABI4 [11], were higher in two OE lines than in the wild-type plants, and the $\mathrm{Na}^{+}$ contents in Arabidopsis shoots and roots were lower in those OE lines as well (Fig. 6b-d). These results were consistent with the repression of $A B I 4$ expression in the transgenic plants constitutively expressing TaBASS2.
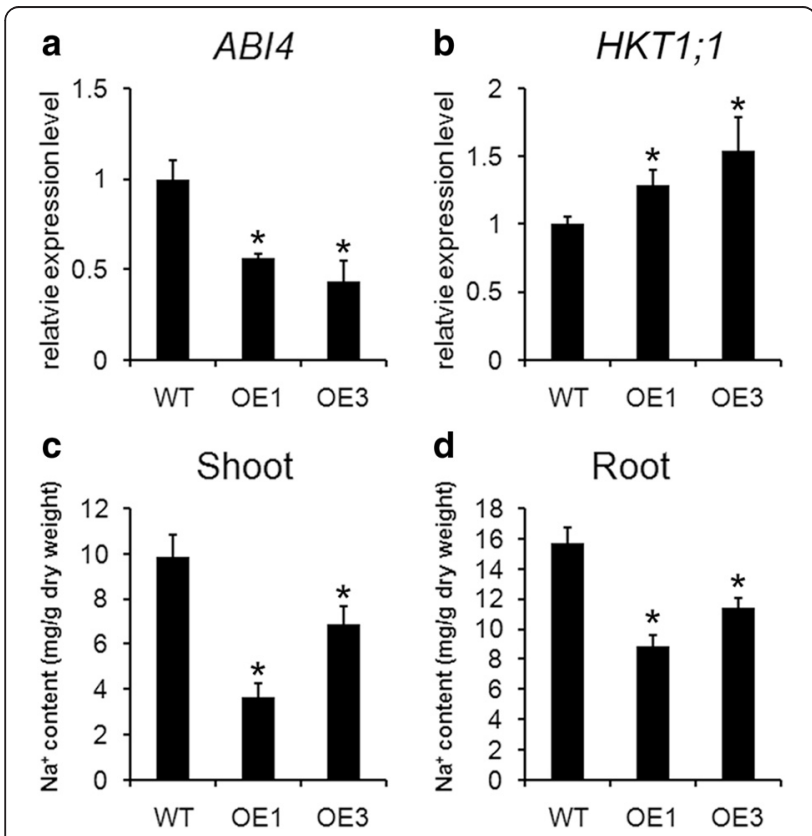

Fig. 6 Constitutively expressing TaBASS2 represses AB/4 expression. a, b The expression levels of $A B I 4$ and $H K T 1 ; 1$ in 12-day-old wild-type and two 35S::TaBASS2 transgenic lines (OE1 and OE3). Error bars represent the standard errors $(n=3)$, with each replicate comprising at least 12 plants. The expression levels were determined by RT-qPCR using AtACT2 in Arabidopsis as the internal control $\mathbf{c}, \mathbf{d}$ The $\mathrm{Na}^{+}$contents in the shoot and root tissue from ten-day-old wild-type and OE lines. Error bars represent the standard errors $(n=3)$, with each replicate comprising at least 200 plants. Columns labeled with an asterisk indicate means differing significantly from the WT result $(P<0.05, t$-test)
As ABI4 was repressed in the OE plants, it was of interest to determine the salinity response when $A B I 4$ remained comparable expression level as in the wildtype. Thus, we carried out a genetic approach to constitutively express $A B I 4$ in $35 S:: T a B A S S 2$ background. Two transgenic lines (TaBASS2OE ABI4OE \#17 from OE1, and TaBASS2OE ABI4OE \#19 from OE3) with ABI4 expression levels comparable as in the wild type were selected for phenotypic assays (Fig. 7f). In comparison with the wild-type, both transgenic lines exhibited a similar response to $\mathrm{NaCl}$ treatment (Fig. 7a-e), and comparable HKT1;1 expression levels (Fig. 7g). These results demonstrated the enhanced resistance conferred by ectopic expression of TaBASS2 vanished when $A B I 4$ expression was restored, indicating that constitutively expressing TaBASS2 in Arabidopsis conferred enhanced salinity tolerance by repressing $A B I 4$ expression.

\section{The transgenic wheat plants overexpressing TaBASS2} have enhanced ROS tolerance and lower $\mathrm{Na}^{+}$contents $\mathrm{DAB}$ staining determined wheat OX lines had higher $\mathrm{H}_{2} \mathrm{O}_{2}$ levels in vivo than the wild-type plants (Fig. 8a). The expression levels of the ROS-scavenging catalase 1 (TaCAT1) were also constitutively up-regulated in the transgenic wheat plants constitutively expressing TaBASS2 (Fig. 8b), along with the CAT1 enzyme activity significantly higher in the transgenic lines (Fig. 8c). These results demonstrated that the constitutive expression of TaBASS2 led to an increase in ROS contents and ROS-scavenging activity in the transgenic wheat plants, suggesting a constitutive activation of ROS signaling. The expression levels of TaHKT1;5-D, the wheat homolog of AtHKT1, were higher in two OX lines than in the wild-type plants (Fig. 8d). The $\mathrm{Na}^{+}$contents in shoots and roots were lower in those OX lines as well (Fig. 8e and $\mathrm{f}$ ). These results suggest that TaHKT1;5-D might be regulated to enhance salinity tolerance in transgenic wheat overexpressing TaBASS2.

\section{Discussion}

Plastid plays a vital role in plant development, stress response, and hormone biosynthesis [24]. Since plasmid harbors its own genome, its function is orchestrated by a combination of anterograde and retrograde signaling [25-27]. The primary ABA biosynthesis pathway takes place in plastid; it starts with the methylerithrytol phosphate (MEP) pathway converting pyruvate to IPP [19]. Bile acid/sodium symporter2 (BASS2) is responsible for the transportation of pyruvate into chloroplast. Knockout of BASS2 in Arabidopsis blocks pyruvate uptake into chloroplast, thus abolishes IPP synthesis in plastid, as evidenced by mevastatin-sensitive phenotype in bass2-1 seedlings [19]. The complementation of bass $2-1$ by constitutive expression of TaBASS2 demonstrates TaBASS2 
a

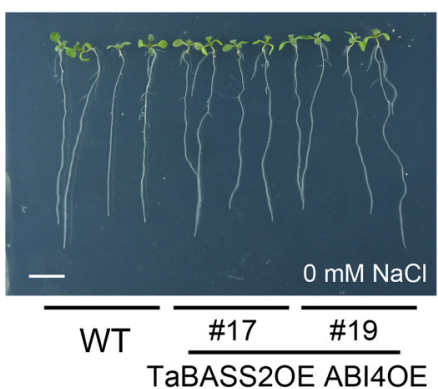

C

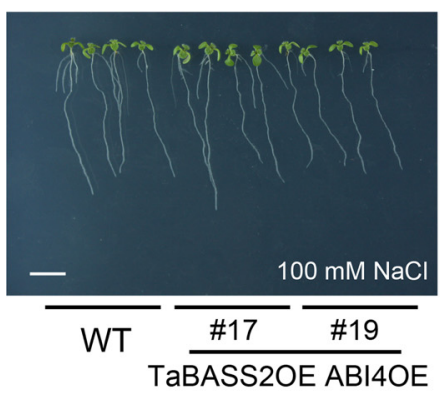

e

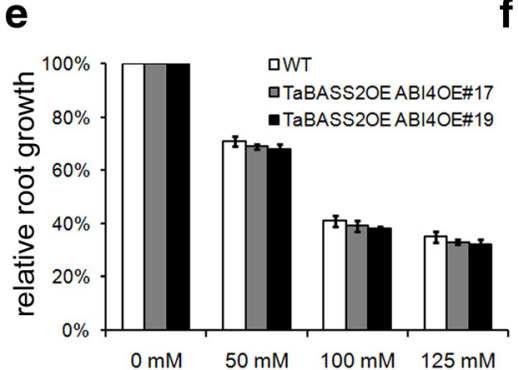

b

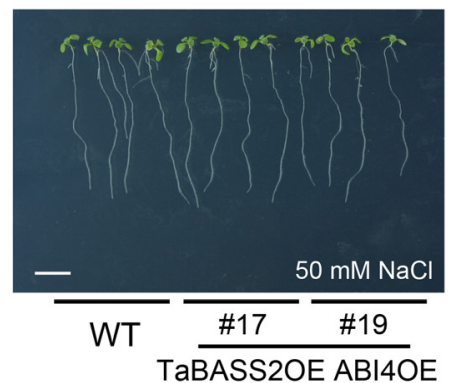

d

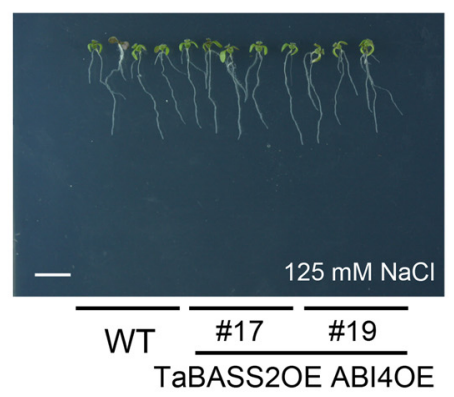

f

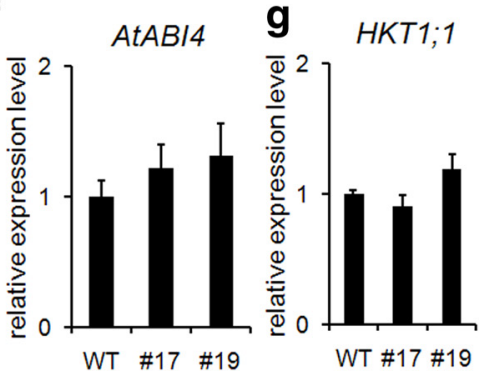

Fig. 7 The enhanced salinity tolerance in 355::TaBASS2 Arabidopsis plants relies on AB/4 suppression. a-d The wild-type seedlings and two transgenic lines constitutively expressing both TaBASS2 and ABI4 (TaBASS2OE ABI4OE \#17 and \#19) after a ten-day treatment with 0, 50, 100 or $125 \mathrm{mM} \mathrm{NaCl}$. Bar $=1 \mathrm{~cm}$. e Relative root growth of the wild-type and TaBASS2OE ABI4OE seedlings. Error bars represent the standard errors $(n=3)$, with each replicate comprising at least 30 seedlings. $\mathbf{f}, \mathbf{g}$ The expression levels of $A B / 4(\mathbf{f})$ and $H K T 1 ; 1(\mathbf{g})$ in the wild-type and TaBASS2OE ABI4OE seedlings. Error bars represent the standard errors $(n=3)$, with each replicate comprising at least 12 seedlings. The expression levels were determined by RT-qPCR using AtACT2 in Arabidopsis as the internal control

functions as a pyruvate transporter in plastid (Fig. 2). Although IPP is the precursor of ABA, its content in the plant tissue was unaffected by the constitutive expression of TaBASS2 (Additional file 7). The marker genes in the ABA signaling pathway remained unchanged as well (Additional file 8a-d). These results established that TaBASS2 did not affect ABA biosynthesis or signaling.

ABI4 has various biological roles in plant development and stress response $[6-9,28]$. Under salinity stress, ABI4 regulates ion homeostasis by its control over the expression of the sodium ion transporter gene AtHKT1;1 [11]. In our 35S::TaBASS2 transgenic Arabidopsis, the expression levels of $A t H K T 1 ; 1$ were constitutively up-regulated (Fig. 6), which is consistent with the previous findings of ABI4 regulating AtHKT1;1 in salt response [11]. Moreover, the enhanced salinity tolerance was disrupted in the transgenic plants with $A B I 4$ expression level restored to the wild-type level (Fig. 7). Hence, the benefit of
TaBASS2 constitutive expression in salinity tolerance depends on its repression of $A B I 4$ expression, which suggests TaBASS2 participates in salt response through regulating $A B I 4$ expression. Because $A B I 4$ homolog has not been identified in wheat, it cannot be tested if TaBASS2 regulates such a signaling node in wheat response to salinity stress. However, we found up-regulation of TaHKT1;5-D, the homolog of AtHKT1;1, as well as reduced $\mathrm{Na}^{+}$concentration in the transgenic wheat seedlings overexpressing TaBASS2 (Fig. 8). These results together suggest a similar mechanism as in Arabidopsis. Besides regulation of $A B I 4$ expression, the constitutive expression of TaBASS2 resulted in increased tissue ROS contents in both wheat and A. thaliana, as did the activity of catalase and the transcription level of its encoding gene CAT1 (Figs. 5 and 8). As a result, the transgenic lines exhibited not just superior salinity tolerance, but also improved tolerance to oxidative stress (Fig. 5, Additional file 6). 
a
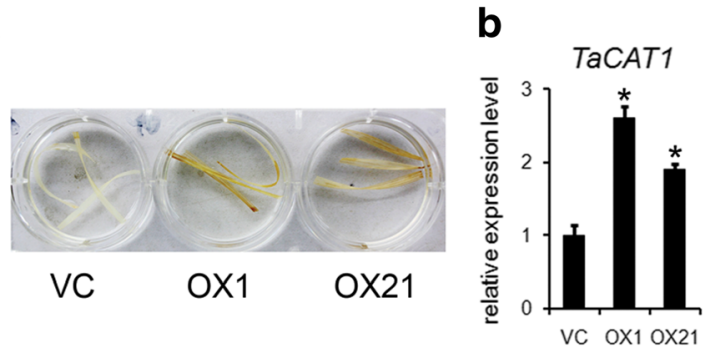

c

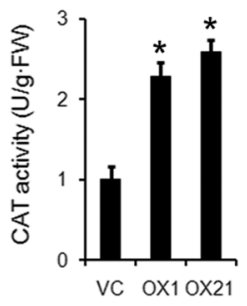

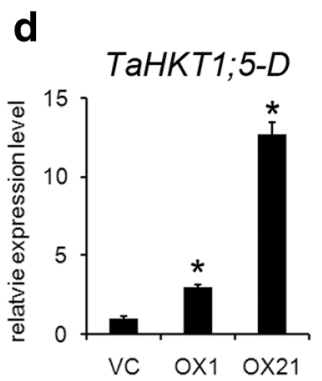
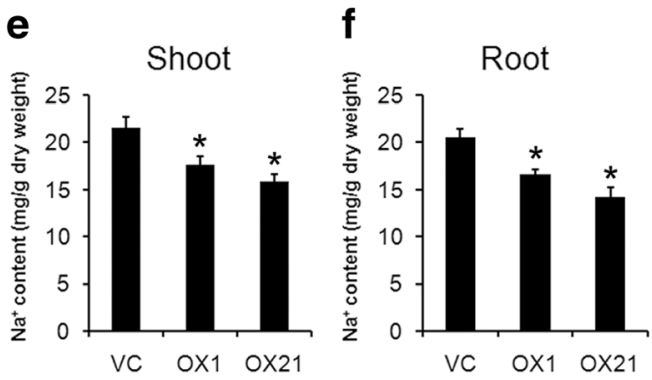

Fig. 8 Constitutively expressing TaBASS2 enhances ROS tolerance and reduces $\mathrm{Na}^{+}$contents in wheat. a DAB staining of the leaves from two-week-old soil-grown vector control (VC) and two Ubi::TaBASS2 lines (OX1 and OX21). b The expression levels of TaCAT1 in two-week-old VC and OX seedlings. c The catalase activity in two-week-old VC and OX seedlings. $\mathbf{d}$ The expression levels of TaHKT1;5-D in two-week-old VC and OX seedlings. Error bars represent the standard errors $(n=3)$, with each replicate comprising at least 12 plants. The expression levels were determined by RT-qPCR using TaCyclophil in in wheat as the internal control. e, $\mathbf{f}$ The $\mathrm{Na}^{+}$contents in the shoot and root tissue from two-week-old VC and OX seedlings. Error bars represent the standard errors $(n=3)$, with each replicate comprising at least 12 plants. Columns labeled with an asterisk indicate means differing significantly from the VC result $(P<0.05$, $t$-test $)$

How TaBASS2, a plastidial pyruvate symporter, functions in salinity tolerance remains an interesting question. The elevated ROS contents in TaBASS2 overexpression plants suggest the involvement of ROS signaling in TaBASS2 function (Fig. 5). Recent studies have shown that ROS not only causes oxidative stress in plant cells, but serves as potential signals in the PET retrograde signaling pathway [16]. ROS accumulation triggers a series of stress-responsive genes, inducing ROS scavenging activity and thereby contributing to the plant's redox homeostasis [8]. Coupled function of RbohD-derived ROS production and plastid hemeoxygenases in salinity response strongly suggests that the chloroplast-to-nucleus retrograde signaling is involved in plant salinity response $[29,30]$. In retrograde signaling pathway, ABI4 serves as a node in the tetrapyrrole and plastid gene expression (PGE). The PGE marker genes LIGHT-HARVESTING CHLOROPHYLL A/ B-BINDING PROTEIN (LHCB) and RUBISCO SMALL SUBUNIT (RBCS) are suppressed by retrograde pathway's activator, norflurazone and lincomycin, while in Arabidposis abi4 mutant these marker genes expression were derepressed [8]. Here, we also found these two genes were less suppressed in 35S::TaBASS2 plants than the wild-type (Additional file 9), indicating that both pathways were affected by the constitutive expression of TaBASS2. The transcription levels of genes in MEcPP pathway, such as MECPP SYNTHASE (MDS) and HYDROPEROXIDE LYASE $(H P L)$, were comparable between the 35S::TaBASS2 and the wild-type plants (Additional file $8 \mathrm{e}$ and $\mathrm{f}$ ). Taken together, these results suggest that ectopic expression of TaBASS2 in Arabidopsis regulates retrograde signaling by repressing $A B I 4$ expression.

\section{Conclusions}

Here in this study, we characterized a putative plastidal pyruvate transporter, TaBASS2, in wheat salinity response. Constitutive expression of TaBASS2 enhanced salinity tolerance in both transgenic wheat and Arabidopsis, accompanied with elevated ROS contents and repression in $A B I 4$ expression. As ROS and ABI4 play crucial roles in plastid-nucleus retrograde signaling, our findings also suggest that TaBASS2 modulates retrograde signaling to positively regulate plant response to salinity stress.

\section{Methods}

\section{Wheat growing conditions and stress treatments}

Two cultivars of bread wheat (Triticum aestivum), Shanrong3 (SR3) and Yangmai20 (YM20), were used in this study. We previously bred SR3 with high salt tolerance from a wheat introgression hybrid, which was constructed with a common wheat cultivar Jinan 177 (with modest salt tolerance) as the recipient and tall wheatgrass (Thinopyrum elongatum, wheat's close relative, one of the monocots with highest salt tolerance) as the donor via asymmetric somatic hybridization 
[21]. YM20, with modest salt tolerance, was bred by Jiangsu Lixiahe Institute of Agricultural Science. Wheat plants were grown in a controlled greenhouse on campus. Wheat plants were grown in half strength Hoagland's liquid medium at $22{ }^{\circ} \mathrm{C}$ under a 16-h-light/ 8 -h-dark photoperiod. For stress treatments, the threeleaf-stage seedlings were treated with $200 \mathrm{mM} \mathrm{NaCl}$, $10 \mathrm{mM} \mathrm{H}_{2} \mathrm{O}_{2}$ or $100 \mu \mathrm{M} \mathrm{ABA}$. The root tissue was harvest after a 48-h treatment for RNA extraction. The phenotypic effects of salinity stress on 10-day-old seedlings were scored after a four-day $\mathrm{NaCl}$ treatment with concentration increasing by $50 \mathrm{mM}$ and another fourday treatment with $200 \mathrm{mM} \mathrm{NaCl}$ treatment, as previously described [31].

Arabidopsis thaliana Columbia-0 (Col-0) is used in this study as the wild-type. The bass2-1 mutant (SALK_101808C) was purchased from Arabidopsis Biological Resource Center. Arabidopsis seeds were surface-sterilized with $70 \%(\mathrm{v} / \mathrm{v})$ ethanol, then placed on Murashige and Skoog (MS) agar plates, which were incubated in the dark at $4{ }^{\circ} \mathrm{C}$ for three days before moved to a $22{ }^{\circ} \mathrm{C}$ growth chamber with a relative humidity of $70 \%$ and a 16-h light/8-h dark photoperiod (light intensity $200 \mu \mathrm{M} \cdot \mathrm{m}^{-2} \cdot \mathrm{s}^{-1}$ ). For stress treatments, four-day-old seedlings were transferred onto a MS plate containing $0,50,100,125 \mathrm{mM} \mathrm{NaCl} ; 0,1,1.5 \mathrm{mM} \mathrm{H}_{2} \mathrm{O}_{2}$; or $0,1,1.5 \mathrm{mM}$ methyl viologen (MV) for ten days. Primary root lengths were measured from digitized images in the Image J software (http://imagej.nih.gov/ij/). Another salinity stress treatment was conducted on the four-weekold soil-grown Arabidopsis plants by adding $50 \mathrm{mM} \mathrm{NaCl}$ for three days, $100 \mathrm{mM}$ for three days, $150 \mathrm{mM}$ for three days and $200 \mathrm{mM}$ for five days, as previously described [32]. The plants were allowed to grow for another two weeks before their survival rates were calculated. For mevastatin treatment, four-day-old seedlings were transferred to the plates supplemented with $0 \mathrm{nM}$ or $500 \mathrm{nM}$ mevastatin and grown vertically for another seven days.

\section{TaBASS2 isolation, sequence characterization and transformation}

The fragment of the TaBASS2 sequence identified in the previous microarray was used to search wheat expression sequence tags (http://www.ncbi.nlm.nih.gov/nucest/ ?term=wheat) [23]. The resulting hits were assembled using CAP3 software [33], and the entire TaBASS2 coding region was cloned using a pair of primers $\left(5^{\prime}\right.$ ATG GCG CCT TCC GCG ACC TGC C-3'/5' -TCA TTC CTT GAA ATC GTC CTT G-3') designed from the reconstructed sequence. The predicted protein sequence was aligned with other BASS2 sequences using the CLUSTALW2 algorithm (www.ebi.ac.uk/Tools/msa/ clustalw2/), and a phylogenetic analysis was conducted using the neighbor-joining method implemented in the
MEGA4 software [34]. To generate transgenic wheat plants, the TaBASS2 coding sequence was ligated into pGA3626 driven by a maize ubiquitin promoter and introduced into wheat cv. YM20 using the shoot apical meristem method [35]. To generate transgenic Arabidopsis, the TaBASS2 coding sequence was ligated into pROK2, and $A B I 4$ into pJIM19 (transgene driven by a $35 \mathrm{~S}$ promoter). The cassette containing TaBASS2 was introduced into either $A$. thaliana ecotype Col-0 or the knockout mutant bass2-1 (SALK_101808C), and the cassette containing the $A B I 4$ coding sequence was introduced into TaBASS2 constitutive expressors using the floral dip method [36]. Transformants were selected as previously [32], and homozygous $\mathrm{T}_{3}$ progeny was used in the subsequent experiments.

\section{Subcellular localization of TaBASS2}

The TaBASS2 coding sequence was inserted into a pBI221 vector with eGFP sequence in frame. TaBASS2-GFP fusion protein was transiently expressed in A. thaliana mesophyll protoplasts using a PEG-mediated transformation method [37]. The protoplasts were then incubated in the dark for $16 \mathrm{~h}$ at $25{ }^{\circ} \mathrm{C}$ before observed under a confocal laser scanning microscopy (Zeiss, Oberkochen, Germany).

\section{Reverse Transcriptase Quantitative PCR}

Total RNA was extracted from plant tissues with the TRIzol reagent (Invitrogen, Carlsbad, CA, USA), followed by cDNA synthesis from $2 \mu \mathrm{g}$ RNA with the SuperScript II reverse transcriptase (Invitrogen). Three biological replicates were included for each assay. Reverse Transcriptase Quantitative PCR (RT-qPCR) was conducted with the FastStart Universal SYBR Green Master (Roche, Basel, Switzerland) on an Eppendorf Mastercycler RT-qPCR device (Eppendorf, Hamburg, Germany). The gene relative expression levels were calculated using the $2^{-\Delta \Delta C T}$ method [38]. A cyclophilin (AF384147) gene and Actin2 (At3g18780) were used as the internal controls in wheat and Arabidopsis, respectively. The RT-qPCR primers are listed in Additional file 10.

\section{Measurement of $\mathrm{Na}^{+}$concentration}

The root and shoot tissues were harvest from the ten-dayold Arabidopsis seedlings grown on MS plates, washed with distilled water for 5 times, dried at $65{ }^{\circ} \mathrm{C}$ for 4 days and digested in $6 \mathrm{M}$ hydrochloric acid solution before the assay, as previously described [39]. The $\mathrm{Na}^{+}$concentrations were determined by a Thermo Iris Intrepid II Inductively Coupled Plasma Atomic Emission Spectrometer (ICP; Thermo Electron Corporation, Franklin, MA). 


\section{Determination of peroxide level, catalase activity and ABA content}

$\mathrm{H}_{2} \mathrm{O}_{2}$ levels in the leaves were determined by the DAB staining of four-week-old soil-grown Arabidopsis plants [40]. Catalase activities were measured in ten-day-old Arabidopsis seedlings with a commercial kit purchased from Beyotime Institute of Biotechnology (Haimen, China). ABA contents in ten-day-old seedlings were assessed as described previously by LC-MS-MS [31].

\section{Ethics}

Not applicable.

\section{Consent to publish}

Not applicable.

\section{Availability of data and materials}

The phylogenetic data shown in Additional file 1b was deposited into a public phylogenetic database, Treebase.org, with the link (http://purl.org/phylo/treebase/phylows/ study/TB2:S19163). The datasets supporting the conclusions of this article are included within the article and its additional files.

\section{Additional files}

Additional file 1: Figure S1. Multi-alignment and phylogenetic analysis of BASS proteins. (a) Multi-alignment of six BASS2 protein sequences. The transmembrane domains (TM1-8) are indicated. The barley HvBASS2 (BAJ88629.1), rice OsBASS2 (NP_917201.1), sorghum SbBASS2 (NP 917201.1), Flaveria trinervia FtBASS2 (BAJ16226.1), and Arabidopsis BASS2 (NP_850089) were used for the multi-alignment. (b) Phylogenetic analysis of 42 BASS proteins. For each sequence, either the gene ID or Gl number was shown. (TIF $236 \mathrm{~kb}$ )

Additional file 2: Figure S2. Subcellular localization of TaBASS2 in Arabidopsis protoplasts. Bar $=10 \mu \mathrm{m}$. (TIF $346 \mathrm{~kb}$ )

Additional file 3: Figure S3. Shoot (a) and root (b) length of 20-dayold wheat vector control (VC) and TaBASS2 overexpression (OX) seedlings. Error bars represent the standard errors $(n=3)$, with each replicate comprising at least 30 plants. Columns labeled with an asterisk indicate means differing significantly from the $V C$ result $(P<0.05$, $t$-test). (TIF $67 \mathrm{~kb}$ )

Additional file 4: Figure S4. The root lengths of the wild-type and two 355::TaBASS2 transgenic lines (OE1 and OE3) after treatment with 0, 50, $100,150 \mathrm{mM} \mathrm{NaCl}$ (a), 0, 1, $1.5 \mathrm{mM} \mathrm{H}_{2} \mathrm{O}_{2}$ (b), or 0, 1, $1.5 \mathrm{mM}$ methyl viologen (MV) (c). (TIF $52 \mathrm{~kb}$ )

Additional file 5: Figure S5. Salinity tolerance in Arabidopsis bass2mutant is comparable with the wild-type plants. (a) Survival rates of four-week-old soil-grown wild-type and bass2-1 plants measured 14 days after $\mathrm{NaCl}$ treatment. Error bars represent the standard errors $(n=3)$, with each replicate comprising at least 30 plants. (b, c) The expression levels of $A B / 4$ (b) and HKT1;1 (c) in 12-day-old wild-type and bass2-1. (TIF 55 kb)

Additional file 6: Figure S6. Constitutively expressing TaBASS2 enhances tolerance to methyl viologen (MV). (a-c) The wild-type seedlings and two 35S:: TaBASS2 transgenic lines (OE1 and OE3) after a ten-day treatment with 0,1 or $1.5 \mathrm{mM} \mathrm{MV}$. Bar $=1 \mathrm{~cm}$. (d) Relative root growth of the wild-type and OE plants treated with 0,1 or $1.5 \mathrm{mM} \mathrm{MV}$. Error bars represent the standard errors $(n=3)$, with each replicate comprising at least 30 plants. Columns labeled with an asterisk indicate means differing significantly from the WT result ( $P<0.05, t$-test). (TIF $2419 \mathrm{~kb}$ )
Additional file 7: Figure S7. The ABA contents of ten-day-old wild-type, two 35S:: TaBASS2 transgenic lines (OE1 and OE3), and bass2-1Arabidopsis seedlings. Error bars represent the standard errors $(n=3)$, with each replicate comprising at least 12 seedlings. (TIF $69 \mathrm{~kb}$ )

Additional file 8: Figure S8. The expression levels of ABA and MECPP markers in ten-day-old seedlings of WT and and two 355.:TaBASS2 transgenic lines (OE1 and OE3). Error bars represent the standard errors $(n=3)$, with each replicate comprising at least 12 seedlings. The expression levels were determined by RT-qPCR using AtACT2 in Arabidopsis as the internal control. (TIF $54 \mathrm{~kb}$ )

Additional file 9: Figure S9. The expression levels of PGE retrograde signaling branch markers, AtRBCS, AtLHCB1.1, and AtLHCB2.4 in three-dayold wild-type and two 35S::TaBASS2 transgenic lines (OE1 and OE3) treated with lincomycin (a) or norflurazone (b). (TIF 72 kb)

Additional file 10: Table S1. PCR primer sequences used in this study. (DOCX $17 \mathrm{~kb})$

Competing interests

The authors declare that they have no competing interests.

\section{Authors' contributions}

$Y Z, X A, M W$ and GX designed the experiments. $Y Z$ and $X A$ carried out most of the experiments. LX quantified hormone contents. $Y Z$ and GX wrote the paper. All authors read and approved the manuscript.

\section{Funding}

This work was supported by grants from the National Basic Research 973 Program of China (2012CB114204), the National Transgenic Project 2013ZX08002003), the Major Program of the Natural Science Foundation of China (No. 31430060) and the Shandong Province Postdoctoral Innovation Project 201202016.

\section{Author details}

${ }^{1}$ The Key Laboratory of Plant Cell Engineering and Germplasm Innovation, Ministry of Education, School of Life Science, Shandong University, 27 Shanda South Road, Jinan, Shandong 250100, China. ${ }^{2}$ Hunan Provincial Key Laboratory of Phytohormones and Growth Development, Hunan Provincial Key Laboratory for Crop Germplasm Innovation and Utilization, Hunan Agricultural University, Changsha 410128, China.

Received: 3 November 2015 Accepted: 29 April 2016

Published online: 10 May 2016

\section{References}

1. Xiong L, Lee $H$, Ishitani M, Zhu JK. Regulation of osmotic stress-responsive gene expression by the LOS6/ABA1 locus in Arabidopsis. J Biol Chem. 2002;277(10):8588-96.

2. Bartels D, Sunkar R. Drought and salt tolerance in plants. Crit Rev Plant Sci. 2005:24(1):23-58.

3. Munns R, Tester M. Mechanisms of salinity tolerance. Annu Rev Plant Biol. 2008:59:651-81

4. Yoshida T, Mogami J, Yamaguchi-Shinozaki K. ABA-dependent and ABA-independent signaling in response to osmotic stress in plants. Curr Opin Plant Biol. 2014;21:133-9.

5. Dinneny JR. Traversing organizational scales in plant salt-stress responses. Curr Opin Plant Biol. 2015;23:70-5.

6. Finkelstein RR, Wang ML, Lynch TJ, Rao S, Goodman HM. The Arabidopsis abscisic acid response locus AB/4 encodes an APETALA 2 domain protein. Plant Cell. 1998;10(6):1043-54.

7. Quesada V, Ponce MR, Micol JL. Genetic analysis of salt-tolerant mutants in Arabidopsis thaliana. Genetics. 2000;154(1):421-36.

8. Koussevitzky S, Nott A, Mockler TC, Hong F, Sachetto-Martins G, Surpin M, Lim J, Mittler R, Chory J. Signals from chloroplasts converge to regulate nuclear gene expression. Science. 2007;316(5825):715-9.

9. Giraud E, Van Aken $\mathrm{O}, \mathrm{Ho} \mathrm{LH}$, Whelan J. The transcription factor ABI4 is a regulator of mitochondrial retrograde expression of ALTERNATIVE OXIDASE1a. Plant Physiol. 2009;150(3):1286-96. 
10. Sun X, Feng P, Xu X, Guo H, Ma J, Chi W, Lin R, Lu C, Zhang L. A chloroplast envelope-bound PHD transcription factor mediates chloroplast signals to the nucleus. Nat Commun. 2011;2:477.

11. Shkolnik-Inbar D, Adler G, Bar-Zvi D. ABI4 downregulates expression of the sodium transporter HKT1;1 in Arabidopsis roots and affects salt tolerance. Plant J. 2013;73(6):993-1005.

12. Mittler R, Vanderauwera S, Gollery M, Van Breusegem F. Reactive oxygen gene network of plants. Trends Plant Sci. 2004;9(10):490-8.

13. Mittler R, Vanderauwera S, Suzuki N, Miller G, Tognetti V, Vandepoele K, Gollery M, Shulaev V, Van Breusegem F. ROS signaling: the new wave? Trends Plant Sci. 2011;16:300-9.

14. Miller G, Suzuki N, Ciftci-Yilmaz S, Mittler R. Reactive oxygen species homeostasis and signalling during drought and salinity stresses. Plant Cell Environ. 2010;33(4):453-67.

15. Zhu JK. Salt and drought stress signal transduction in plants. Annu Rev Plant Biol. 2002;53:247-73.

16. Kim C, Apel K. ${ }^{1} \mathrm{O}_{2}$-mediated and EXECUTER-dependent retrograde plastid-to-nucleus signaling in norflurazon-treated seedlings of Arabidopsis thaliana. Mol Plant. 2013;6(5):1580-91.

17. Baruah A, Simkova K, Hincha DK, Apel K, Laloi C. Modulation of ${ }^{1} \mathrm{O}_{2}$ mediated retrograde signaling by the PLEIOTROPIC RESPONSE LOCUS 1 (PRL1) protein, a central integrator of stress and energy signaling. Plant J. 2009;60(1):22-32

18. Kindgren P, Kremnev D, Blanco NE, de Dios Barajas Lopez J, Fernandez AP, Tellgren-Roth C, Kleine T, Small I, Strand A. The plastid redox insensitive 2 mutant of Arabidopsis is impaired in PEP activity and high light-dependent plastid redox signalling to the nucleus. Plant J. 2012;70(2):279-91.

19. Furumoto T, Yamaguchi T, Ohshima-Ichie $Y$, Nakamura M, Tsuchida-Iwata $Y$, Shimamura M, Ohnishi J, Hata S, Gowik U, Westhoff P, et al. A plastidial sodium-dependent pyruvate transporter. Nature. 2011;476(7361):472-5.

20. Xia GM, Xiang FN, Zhou AF, Wang HA, Chen HM. Asymmetric somatic hybridization between wheat (Triticum aestivum L.) and Agropyron elongatum (Host) Nevishi. Theor Appl Genet. 2003;107(2):299-305.

21. Peng Z, Wang M, Li F, LV H, Li C, Xia G. A proteomic study of the response to salinity and drought stress in an introgression strain of bread wheat. Mol Cell Proteomics. 2009;8(12):2676-86.

22. Xia GM. Progress of chromosome engineering mediated by asymmetric somatic hybridization. J Genet Genomics. 2009;36(9):547-56.

23. Liu C, Li S, Wang M, Xia G. A transcriptomic analysis reveals the nature of salinity tolerance of a wheat introgression line. Plant Mol Biol. 2012;78(1-2):159-69

24. Demarsy E, Buhr F, Lambert E, Lerbs-Mache S. Characterization of the plastid-specific germination and seedling establishment transcriptional programme. J Exp Bot. 2012;63(2):925-39.

25. Pogson BJ, Woo NS, Forster B, Small ID. Plastid signalling to the nucleus and beyond. Trends Plant Sci. 2008;13(11):602-9.

26. Woodson JD, Chory J. Coordination of gene expression between organellar and nuclear genomes. Nature. 2008;9:38301-13.

27. Jung HS, Chory J. Signaling between chloroplasts and the nucleus: can a systems biology approach bring clarity to a complex and highly regulated pathway? Plant Physiol. 2010;152:453-9.

28. Leon P, Gregorio J, Cordoba E. ABI4 and its role in chloroplast retrograde communication. Front Plant Sci. 2013:3:304.

29. Xie YJ, Xu S, Han B, Wu MZ, Yuan XX, Han Y, Gu Q, Xu DK, Yang Q, Shen WB. Evidence of Arabidopsis salt acclimation induced by up-regulation of $\mathrm{HY} 1$ and the regulatory role of RbohD-derived reactive oxygen species synthesis. Plant J. 2011;66(2):280-92.

30. Golldack D, Li C, Mohan H, Probst N. Tolerance to drought and salt stress in plants: unraveling the signaling networks. Front Plant Sci. 2014;5:151.

31. Dong W, Wang M, Xu F, Quan T, Peng K, Xiao L, Xia G. Wheat oxophytodienoate reductase gene TaOPR 1 confers salinity tolerance via enhancement of abscisic acid signaling and reactive oxygen species scavenging. Plant Physiol. 2013;161(3):1217-28.

32. Zhao Y, Dong W, Zhang N, Ai X, Wang M, Huang Z, Xiao L, Xia G. A wheat allene oxide cyclase gene enhances salinity tolerance via jasmonate signaling. Plant Physiol. 2013;164(2):1068-76.

33. Huang X, Madan A. CAP3: A DNA sequence assembly program. Genome Res. 1999:9:868-77.

34. Kumar S, Tamura K, Nei M. MEGA3: Integrated software for Molecular Evolutionary Genetics Analysis and sequence alignment. Brief Bioinformatics. 2004;5:150-63.
35. Zhao TJ, Zhao SY, Chen HM, Zhao QZ, Hu ZM, Hou BK, Xia GM. Transgenic wheat progeny resistant to powdery mildew generated by Agrobacterium inoculum to the basal portion of wheat seedling. Plant Cell Rep. 2006; 25(11):1199-204.

36. Clough SJ, Bent AF. Floral dip: a simplified method for Agrobacteriummediated transformation of Arabidopsis thaliana. Plant J. 1998;16(6):735-43.

37. Yoo S-D, Cho Y-H, Sheen J. Arabidopsis mesophyll protoplasts: a versatile cell system for transient gene expression analysis. Nat Protoc. 2007;2:1565-72.

38. Livak K, Schmittgen T. Analysis of relative gene expression data using real-time quantitative PCR and the $2^{-\Delta \Delta C T}$ method. Methods. 2001;25:402-8.

39. Pandey GK, Cheong YH, Kim BG, Grant JJ, Li L, Luan S. CIPK9: a calcium sensor-interacting protein kinase required for low-potassium tolerance in Arabidopsis. Cell Res. 2007;17(5):411-21.

40. Asselbergh B, Curvers K, França S, Audenaert K, Vuylsteke M, Van Breusegem F. Höfte M. Resistance to Botrytis cinerea in sitiens, an abscisic acid-deficient tomato mutant, involves timely production of hydrogen peroxide and cell wall modifications in the epidermis. Plant Physiol. 2007;144:1863-77.

\section{Submit your next manuscript to BioMed Central and we will help you at every step:}

- We accept pre-submission inquiries

- Our selector tool helps you to find the most relevant journal

- We provide round the clock customer support

- Convenient online submission

- Thorough peer review

- Inclusion in PubMed and all major indexing services

- Maximum visibility for your research

Submit your manuscript at www.biomedcentral.com/submit

) Biomed Central 